\title{
Dendritic cells vaccine: the basics and selected applications in cancer
}

\begin{abstract}
Dendritic cells vaccine (DCV) is a newly emerging and potent form of immune therapy used to treat cancer. DCV enhances the circumvented immune system by educating and reprogramming it to mount a specific immune response against the target. Although this vaccination approach has been effective and feasible, optimal techniques for other aspects of vaccine production are still in the experimental stage. The basic aspects of dendritic cell (DC) biology and functions, along with the DCV preparation process and selected applications are discussed here
\end{abstract}

Keywords: immunotherapy, antigen, vaccines, cancer, cell therapy
Volume 2 Issue 3 - 2015

\author{
Atlal Abusanad \\ Internal medicine Department, King Abdulaziz University, Saudi \\ Arabia \\ Correspondence: Atlal Abusanad, Division of Medical \\ oncology, Internal Medicine Department, King, Abdulaziz \\ university Hospital, Al Sulimanyiah district, P.O. Box 80215, \\ Jeddah 21589, Saudi Arabia, Tel +966555016860, \\ Email atlal.abusanad@mail.mcgill.ca,AAbusanad@Kau.edu.sa
}

Received: January 08, 2015 | Published: March 31, 2015
Abbreviations: DC, dendritic cells; DCV, dendritic cell vaccine; PBMCs, peripheral blood mononuclear cells; PAMPS, pathogen-associated molecular patterns; TAA, tumor-associated antigen; MHC, major histo-compatibility; TLRs, toll-like receptors; NK, natural killer cells

\section{Introduction}

Since the discovery of dendritic cells (DC) four decades ago, ${ }^{1}$ their structure, location, and central role in regulating the immune response have been well elucidated. DCs are highly specialized cells involved in host immune defense against an array of antigenic responses, such as infections of viruses and bacteria, and malignancy. DCs represent an attractive target for immuno-modulation, particularly in diseases such as cancer and HIV infection. The unique qualities and role of DCs in triggering an immune response, combined with the growing evidence that the immune system can attack and eradicate malignant cells and certain chronic infections, form the basis for dendritic cell vaccine $(\mathrm{DCV}){ }^{2}$

\section{Overview of dendritic cells}

Dendritic cells originate from the bone marrow and represent an extremely heterogeneous population with several subsets defined by their unique cell surface antigens and location. The subsets fall into two main types, myeloid and plasma cytoid, which are derived from monocytes and lymphocytes, respectively. ${ }^{3}$ Peripheral blood mononuclear cells (PBMCs) have the potential to give rise to DCs under certain conditions, when exposed to pathogen-associated molecular patterns (PAMPS), such as viruses, bacterial lipopolysaccharide (LPS), flagellin, or tumor-associated antigen (TAA), which results in a cytokine-mediated inflammatory response. A small number of DCs are released into the circulation in the immature form (iDC) with the ability to mature in the same fashion as triggered PBMCs.

The DC journey starts once they are released into the circulation, from where they may relocate to a specific tissue or be transported freely in the blood. Some reside in tissue exposed to the exterior environment, functioning as a surveillance apparatus, e.g., Langerhans cells in the skin, where they can be challenged and constantly triggered by the surrounding environment (Figure 1). Similarly, DCs are abundant in the mucosal surfaces of the intestine and airways.
Foreign antigen is taken up by the $\mathrm{DC}$, processed, combined with major histo-compatibility (MHC) class II, which, along with selfantigen combined with MHC class I, is presented on the cell surface. They migrate to site-draining lymph nodes and related lymphoid structures guided by chemokine gradients, to present the captured antigen to other cells of the immune system. ${ }^{4}$ The maturation of DCs is influenced by an array of inflammatory cytokines. Such maturation closely correlates with the nature of the offensive signal. ${ }^{5}$

Dendritic cells play a central role in orchestrating the immune response because they:

a. Express both MHC class I and II molecules, alongside several different co stimulatory receptors [B7-1 (CD80), B7-2 (CD86), CD40 receptor, and $\mathrm{CD} 1 \mathrm{a}]$, adhesion molecules [ICAM-1 (CD54) and LFA-3 (CD58)], and toll-like receptors (TLRs), which on binding with foreign antigen and ligands expressed by other immune cells, together activate DCs, initiating an immune response. ${ }^{6}$

b. Are highly proficient and potent antigen-presenting cells that capture, process, and present exogenous and endogenous antigen to cells of both the innate (naïve T lymphocytes: T helper 1 [Th1] cells, cytotoxic T lymphocytes [CTL] and natural killer [NK] cells) and the adaptive (B lymphocytes) immune systems.?

c. Produce autocrine and paracrine cytokines upon activation, which further facilitate their maturation and specialization. ${ }^{8}$

d. Possess unique morphology with many finger-like projections that facilitate migration to the lymph nodes, and specialized lymphoid structures for efficient antigen presentation. ${ }^{3}$

\section{Dendritic cells vaccine}

Dendritic cells vaccine is an active immunotherapy in which antigen-loaded autologous DCs are used to induce an immune response against a specific target. Vaccine production is a multistep process (Figure 2).

\section{Dendritic cells vaccine preparation}

Obtaining dendritic cells: Arelatively large number of DCs is required to produce effective vaccines. These are obtained as immature dendritic 
cells (iDC) from various sources, CD34+ hematopoietic progenitor cells, or CD14+ monocytes precursors. ${ }^{9}$ Circulating iDCs comprise $0.5 \%$ of PBMCs and can be isolated from $\mathrm{T}$ cell- and monocytedepleted peripheral blood cells after culturing for 48 hours in vitro in the absence of cytokines. While in culture, iDCs become less dense, which facilitate their purification by density-gradient centrifugation. ${ }^{10}$ Leukapharesis and immuno-phenotyping were used to isolate CD14+ monocytes and blood $\mathrm{CD} 34+$ progenitors. ${ }^{11,12}$

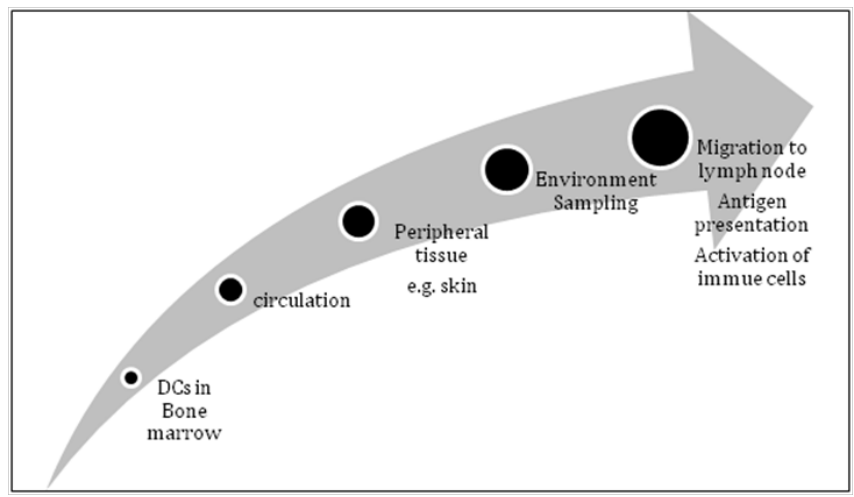

Figure I life cycle of dendritic cells (DCs) from bone marrow to periphera tissue via circulation where they encounter different Antigens. In the draining lymph node/lymphoid structure, they present captured antigen(s) and interact with other immune cells.

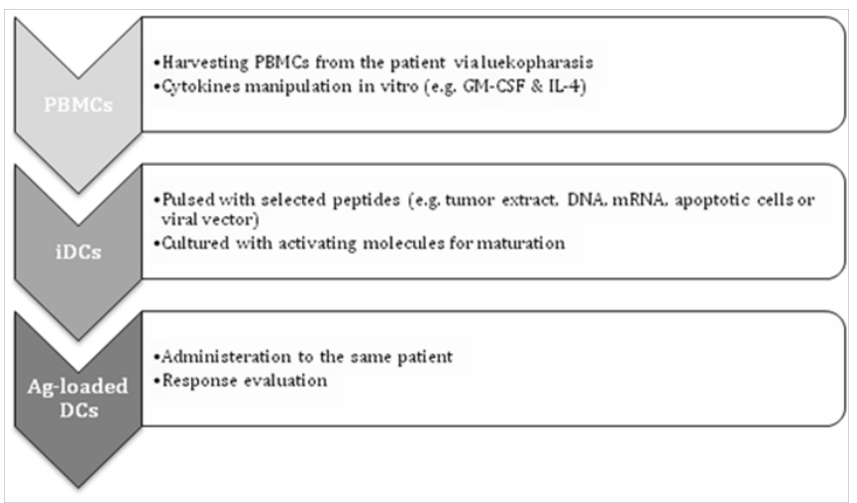

Figure 2 Dendritic cells vaccine preparation process.

PBMCs, peripheral blood mononuclear cells; iDCs, immature dendritic cells; $\mathrm{Ag}$, antigen

Culturing CD34+ hematopoietic cells or CD14+ monocytes, with a variety of different cytokines in vitro, can generate adequate numbers of DCs. Sallusto et al., ${ }^{13}$ were the first to describe the generation of DCs in vitro from CD14+ monocytes after 5-7 days incubation with granulocyte-macrophage colony-stimulating factor (GM-CSF) and IL-4. CD34+ progenitor cells cultured with a combination of GM-CSF and TNF, mature into fully functional DC- like cells. ${ }^{14}$ The generated cells acquired stable morphologic and functional characteristics of DCs.

In order to minimize the ex vivo manipulation of DCs, and to reduce the risk of contamination, Flt3 ligand was administered in vivo to induce cellular mobilization. Flt3 ligand binds to its tyrosinekinase receptor (RTK) Flt3 (CD135), which is expressed on early hematopoietic cells, and affects their proliferation. Stimulation of Flt3/CD135 causes an increase in peripheral blood DCs. ${ }^{15}$ While the optimal method of generating fully functional DCs has not yet been identified, CD14+ monocyte- and CD34+ progenitor-derived DCs are the most widely used and clinically successful.

\section{Antigen selection and loading}

A principal element of a successful DCV is identification and selection of the most appropriate antigens. Appropriate antigens for loading DCs are specific, immunogenic, MHC class I- and/or II-avid, stable, and sufficiently expressed by the target. They should also retain their immunogenicity in vivo.

Loading DCs with peptide derived from a known antigen is the most common strategy. Other strategies are the use of whole cells (e.g., apoptotic tumor cells, allogeneic tumor cell lines, and cell lysates), recombinant proteins, immune complexes, and antibodies directed specifically toward DC molecules. Whole cell extracts can provoke a non-specific immune response, but they can also increase the likelihood of immunogenicity because of the presence of polyvalent peptides within the processed cell. However, this approach is less useful in proof-of-principle studies and presentation of selfpeptides can result in autoimmunity. In addition, processing such complex antigen could be difficult for DCs with resultant suboptimal antigen presentation. On the contrary, antigen-derived peptide/epitope with or without conjugate may trigger a specific immune response with a reduced risk of autoimmunity but diminished immunogenicity. The limited number of well-characterized antigens and the relatively rapid turnover of exogenous peptides are other significant limitations. The production of an engineered multi-epitope antigen, in an attempt to maximize immunogenicity and specificity while minimizing autoimmunity, may help to overcome these limitations.

Antigen loading strategies such as transducing DCs with a viral vector and transfecting DCs with RNA or plasmid DNA have been tested successfully in animal models. ${ }^{16,17}$ The goal is to enable a steady and continuous expression of endogenous tumor antigens by DCs, and allow presentation of multiple epitopes with concurrent presentation of both MHC class I- and II-restricted epitopes. However, antigen expression in genetically engineered DCs depends on the transfection efficiency, which is generally low, ranging from $10-20 \%$. Antigen loading techniques can differ according to the type of antigen used in DC priming, but the general practice is to add antigens to DCs in culture and incubate for several days, to enable their recognition and presentation by DCs.

\section{Induction of dendritic cells maturation}

Dendritic cells maturation is a process whereby the iDC undergoes several morphologic and functional modifications to become proficient at antigen presentation. It involves the loss of phagocytic and endocytic receptors, upregulation of MHC class II and costimulatory/adhesion molecules (CD40, CD50, CD54, CD58, CD80, and CD86), expression of CD83 and chemokine receptor 7 [CCR7] (molecules that are essential for DC migration) and attainment of the characteristic star-like morphology. The mature DC has the capability of producing IL-12, which is required for the development of Th1 cells and the CTL response. In vivo, maturation occurs while DCs are migrating toward the draining lymph node, and is initiated through the activation of TLRs. ${ }^{18}$ In vitro, a combination of cytokines is used to induce a comparable process. A combination of pro-inflammatory cytokines such as IL-1, TNF- $\beta$, IL-6, and prostaglandin E2 was employed and resulted in DCs capable of eliciting potent Th1 cell and CTL responses. ${ }^{19}$ Culture of iDCs with such as combination of cytokines is currently the gold standard; however, others have also explored TLR-dependent (e.g., LPS, receptor-directed monoclonal antibodies, and immune complexes) and TLR- independent (e.g., RTK stimulation, transfection of RNA/DNA, and exosomes) approaches to induce DC maturation. ${ }^{20}$ 


\section{Delivery to the host}

Route: As with many aspects of the developmental process of $\mathrm{DCV}$, the optimal route of administration has yet to be identified. Administration routes include intravenous, intradermal, and to a lesser extent intraperitoneal, or directly into the lymphatic vessels and/or lymph nodes. ${ }^{21}$ Each has its own rationale; intradermal and intravenous routes provide convenience as they are easy to access, but large numbers of DCs are required to ensure that an adequate number reach the lymph nodes to yield the desired effect. The intralymphatic/ nodal route provides direct access to the microenvironment where antigen presentation takes place, hence fewer DCs are needed in comparison to other routes, but the need for radiological-guided administration makes it less preferable.

Behind the scenes (migration to the lymph node): The motility and the ability of DCs to migrate toward the lymph node increase with activation and maturation. The migration process is enabled by gradients of chemokines that attract DCs to the site of action. iDCs respond to many chemokines (MIP-1a, MIP-1b, MIP-3a, MIP-5, MCP-3, MCP-4, RANTES, TECK, and SDF-1), which are inducible upon inflammatory stimulation. Mature DCs upregulate CCR7 during activation that has a vital role in the process of migration through the binding of its ligands, CCL19 and CCL21. The latter are produced primarily in the T cell- rich parafollicular areas of the lymph nodes. ${ }^{22}$ Enriching DC with TNF- $\alpha$ is suggested to optimize the migration process. ${ }^{23}$ However, little is known about how to optimize DC migration and research is ongoing to optimize DCV delivery.

\section{Response to dendritic cell vaccines}

Two interactions have been proposed as mechanisms for the antitumor effect of DC-based immunotherapy. The first involves the delivery of DCs directly to the tumor site or the migration of DCs from the vaccine-draining lymph node to the tumor. In the tumor, activated DCs pick up available antigens and produce cytokines creating a pro- inflammatory response, and several antigens are processed and presented by DCs to T cells leading to 'antigen/epitope spreading' (an amplified immune response but with relatively low specificity for the vaccine-associated antigen). This response targets the tumor in general. Secondly, DC therapies induce both $\mathrm{T}$ cell- mediated helper and cytolytic responses specific for epitopes presented by the transferred DCs. In this model, the anti-tumor effect is mediated via targeting specific TAA., ${ }^{2,3}$

The capability of DCVs to elicit an immune response in vitro is evaluated with several quantitative and qualitative assays. Cytokine and chemokine production and receptor expression by DCs are measured to assess how mature and competent they are (e.g., IL-12 production is indicative of maturation and CCR7 receptor expression is indicative of migratory capacity). The ability to trigger an immune response beyond a defined threshold is assessed by quantifying the stimulated cells of the immune system (e.g., CD8+ T cells and/or CD4+ T cells). ${ }^{24}$ In vivo, stimulated $\mathrm{T}$ cells in the peripheral blood can be easily accessed for this kind of assessment. However, there is a variable correlation between vaccine parameters and clinical response to vaccination that may be explained in general by the diversity of the host's immune system, the extent of disease, and the negative immuno modulatory effect of the disease, whether cancer or chronic infection. Disease extent is a recognized factor in predicating the clinical response to DCV; the greater the disease burden, the lower the efficacy. Current evidence, therefore, suggests that vaccination may be best carried out in the adjuvant setting following primary surgery or chemotherapy, when host tumor burden is minimal.
In vivo, a significant obstacle to the success of DC-based immunotherapy is the presence of negative immunomodulation mediated by regulatory T cells, NK cells, NKT cells, and an array of immunosuppressive cytokines (e.g., IL4, IL-6, IL-10, and IFN- $\gamma$ ). Several other mechanisms from the tumor microenvironment inhibit DCs to induce an efficient anti-tumor response. Tumor-derived factors such as VEGF, TGF- $\beta$, M-CSF, GM-CSF, IL- 6 , and IL-10 affect DC functions. For example, TGF- $\beta$ results in the impairment of DC function, thereby resulting in an accumulation of myeloid-derived suppressor cells, and detrimental M2 macrophages. Down-regulation of such inhibitory immune mechanisms can boost the effect of DCV. In animal models, low-dose cyclophosphamide and depletion of $\mathrm{T}$ regulatory (T-reg) and NK cells enhanced the anti-tumor effect of $\mathrm{DCV}^{25}$

\section{Selected applications of DCV in cancer treatment}

Sipuleucel- $\mathrm{T}$ is the first approved DCV for patients with asymptomatic or minimally symptomatic metastatic castrationresistant prostate cancer. It is directed against a recombinant antigen (PAP-GM-CSF) that consists of prostatic acid phosphatase (PAP), an antigen expressed in more than $95 \%$ of prostate cancers, and GM-CSF, an immune cell activator. It showed significant survival advantage compared to placebo in a phase III trial. ${ }^{26}$ Certain hematological malignancies such as B cell non-Hodgkin's lymphomas and multiple myeloma express monoclonal immunoglobulin, which is a readily targetable antigen. DCV directed against the antigenic determinant of immunoglobulin, known as the idiotype (Id) has been used in small clinical trials. ${ }^{27-29}$ Several melanoma antigens have been identified that bind MHC class I. Among these are gp100, MART-1, tyrosinase, MAGE-1, and MAGE-3. Several early-phase trials showed DCVs against these antigens to be feasible and of therapeutic benefit; however, there is a paucity of information regarding their use in everyday clinical practice..$^{30,31}$

\section{Conclusion}

Dendritic cells are proficient antigen-presenting cells, which have been associated with immunogenicity and tolerogenicity to various tumor- and infection-derived antigens. ${ }^{32,33}$ They mediate and coordinate the interaction between both innate and adaptive immune responses through their ability to present foreign antigens to different immune cells, ${ }^{7}$ and possess certain molecular and morphological features that facilitate their key role. DCs work in harmony with other mediators of the immune system and require various cytokines and chemokines to mediate their maturation and migration, and presentation of the offensive antigen. ${ }^{6,18,22}$

DCs are ideal for immunotherapy in cancer patients. DCVs are made by PBMCs such as lymphocytes or monocytes, harvested from the blood of the patient and grown ex vivo in large amounts while exposing them to antigens (e.g., from the patient's own cancer). ${ }^{11,12,24}$ The DC cells with antigens presented on their surface are injected back into the patient. The injected DCs along with the antigens activate the production of CTL and others immune cells, which are programmed to recognize and destroy the cancer cells. ${ }^{3}$ Several DCVs have been in phase II and III clinical trials. ${ }^{16,26-29}$ Important considerations in designing DCV trials are: selecting the appropriate antigen, choosing the appropriate cytokine profile to achieve the desired outcome, deciding on the route of administration, and using combinations of treatment modalities. Our knowledge on DC as a valuable tool for exploiting human immunity against diseases in general and cancer in particular is growing apace will be useful in the research for further advances in cancer research. 


\section{Acknowledgments}

The author thanks Ms. Lindsey Goff for editing the final draft of this manuscript.

\section{Conflicts of interest}

Author declares that there is no conflict of interest.

\section{References}

1. Steinman RM, Cohn ZA. Identification of a novel cell type in peripheral lymphoid organs of mice. I. Morphology, quantitation, tissue distribution. J Exp Med. 1973;137(5):1142-1162.

2. Ardavin C, Amigorena S, Reis e Sousa C. Dendritic cells: immunobiology and cancer immunotherapy. Immunity. 2004;20(1):17-23.

3. Lipscomb MF, Masten BJ. Dendritic cells: immune regulators in health and disease. Physiol Rev. 2002;82(1):97-130.

4. Granucci F, Zanoni I. The dendritic cell life cycle. Cell Cycle. 2009;8(23):3816-3821.

5. Satthaporn S, Eremin O. Dendritic cells (I): Biological functions. $J R$ Coll Surg Edinb. 2001;46(1):9-19.

6. Stockwin LH, McGonagle D, Martin IG, et al. Dendritic cells: immunological sentinels with a central role in health and disease. Immunol Cell Biol. 2000;78(2):91-102.

7. Palucka K, Banchereau J. Dendritic cells: a link between innate and adaptive immunity. J Clin Immunol. 1999;19(1):12-25.

8. de Saint-Vis B, Fugier-Vivier I, Massacrier C, et al. The cytokine profile expressed by human dendritic cells is dependent on cell subtype and mode of activation. J Immunol. 1998;160(4):1666-1676.

9. Alejandro Lopez J, Crosbie G, Kelly C, et al. Monitoring and isolation of blood dendritic cells from apheresis products in healthy individuals: a platform for cancer immunotherapy. $J$ Immunol Methods. 2002;267(2):199-212

10. Freudenthal PS, Steinman RM. The distinct surface of human blood dendritic cells, as observed after an improved isolation method. Proceedings of the National Academy of Sciences of the United States of America. 1990;87(19):7698-7702.

11. Babatz J, Rollig C, Oelschlagel U, et al. Large-scale immunomagnetic selection of CD14+ monocytes to generate dendritic cells for cancer immunotherapy: a phase I study. Journal of Hematotherapy \& Stem Cell Research. 2003;12(5):515-523.

12. Siena S, Di Nicola M, Bregni M, et al. Massive ex vivo generation of functional dendritic cells from mobilized CD34+ blood progenitors for anticancer therapy. Exp Hematol. 1995;23(14):1463-1471.

13. Sallusto F, Lanzavecchia A. Efficient presentation of soluble antigen by cultured human dendritic cells is maintained by granulocyte/macrophage colony-stimulating factor plus interleukin 4 and down regulated by tumor necrosis factor alpha. J Exp Med. 1994;179(4):1109-1118.

14. Shortman K, Caux C. Dendritic cell development: multiple pathways to nature's adjuvants. Stem Cells. 1997;5(6):409-419.

15. Harada S, Kimura T, Fujiki H, et al. Flt3 ligand promotes myeloid dendritic cell differentiation of human hematopoietic progenitor cells: possible application for cancer immunotherapy. Int $J$ Oncol. 2007;30(6):1461-1468
16. Mu LJ, Kyte JA, Kvalheim G, et al. Immunotherapy with allotumour mRNA-transfected dendritic cells in androgen-resistant prostate cancer patients. Br J Cancer. 2005;93(7):749-756.

17. Mitchell DA, Nair SK. RNA-transfected dendritic cells in cancer immunotherapy. The Journal of Clinical Investigation. 2000;106(9):1065-1069.

18. Park J, Babensee JE. Differential functional effects of biomaterials on dendritic cell maturation. Acta Biomater. 2012;8(10):3606-3617.

19. Steinbrink K, Paragnik L, Jonuleit H, et al. Induction of dendritic cell maturation and modulation of dendritic cell-induced immune responses by prostaglandins. Arch Dermatol Res. 2000;292(9):437-445.

20. Li DY, Gu C, Min J, et al. Maturation induction of human peripheral blood mononuclear cell-derived dendritic cells. Exp Ther Med. 2012;4(1):131-134.

21. Lesterhuis WJ, de Vries IJ, Schreibelt G, et al. Route of administration modulates the induction of dendritic cell vaccine-induced antigenspecific $\mathrm{T}$ cells in advanced melanoma patients. Clin Cancer Res. 2011;17(17):5725-5735.

22. Martin-Fontecha A, Lanzavecchia A, Sallusto F. Dendritic cell migration to peripheral lymph nodes. Handb Exp Pharmacol. 2009;2009(188):31-49.

23. Yrlid U, Milling SW, Miller JL, et al. Regulation of intestinal dendritic cell migration and activation by plasmacytoid dendritic cells, TNFalpha and type 1 IFNs after feeding a TLR7/8 ligand. J Immunol. 2006;176(9):5205-5212.

24. Vopenkova K, Mollova K, Buresova I, et al. Complex evaluation of human monocyte-derived dendritic cells for cancer immunotherapy. $J$ Cell Mol Med. 2012;16(11):2827-2837.

25. Dang Y, Wagner WM, Gad E, et al. Dendritic cell-activating vaccine adjuvants differ in the ability to elicit antitumor immunity due to an adjuvant-specific induction of immunosuppressive cells. Clin Cancer Res. 2012;18(11):3122-3131.

26. Kantoff PW, Higano CS, Shore ND, et al. Sipuleucel-T immunotherapy for castration-resistant prostate cancer. N Engl J Med. 2010;363(5):411-422.

27. Di Nicola M, Zappasodi R, Carlo-Stella C, et al. Vaccination with autologous tumor-loaded dendritic cells induces clinical and immunologic responses in indolent B-cell lymphoma patients with relapsed and measurable disease: a pilot study. Blood. 2009;113(1):18-27.

28. Timmerman JM, Czerwinski DK, Davis TA, et al. Idiotype-pulsed dendritic cell vaccination for B-cell lymphoma: clinical and immune responses in 35 patients. Blood. 2002;99(5):1517-1526

29. Hsu FJ, Caspar CB, Czerwinski D, et al. Tumor-specific idiotype vaccines in the treatment of patients with B-cell lymphoma-long-term results of a clinical trial. Blood. 1997;89(9):3129-3135.

30. Banchereau J, Palucka AK, Dhodapkar M, et al. Immune and clinical responses in patients with metastatic melanoma to $\mathrm{CD} 34(+)$ progenitorderived dendritic cell vaccine. Cancer Res. 2001;61(17):6451-6458.

31. Baldueva IA, Novik AV, Moiseenko VM, et al. [Phase II clinical trial of autologous dendritic cell vaccine with immunologic adjuvant in cutaneous melanoma patients]. Vopr Onkol. 2012;58(2):212-221.

32. Steinman RM. The dendritic cell system and its role in immunogenicity. Annu Rev Immunol. 1991;9:271-296.

33. Nestle F. Dendritic cells vaccination for cancer therapy. Oncogene. 2000;19(56):6673-6679. 